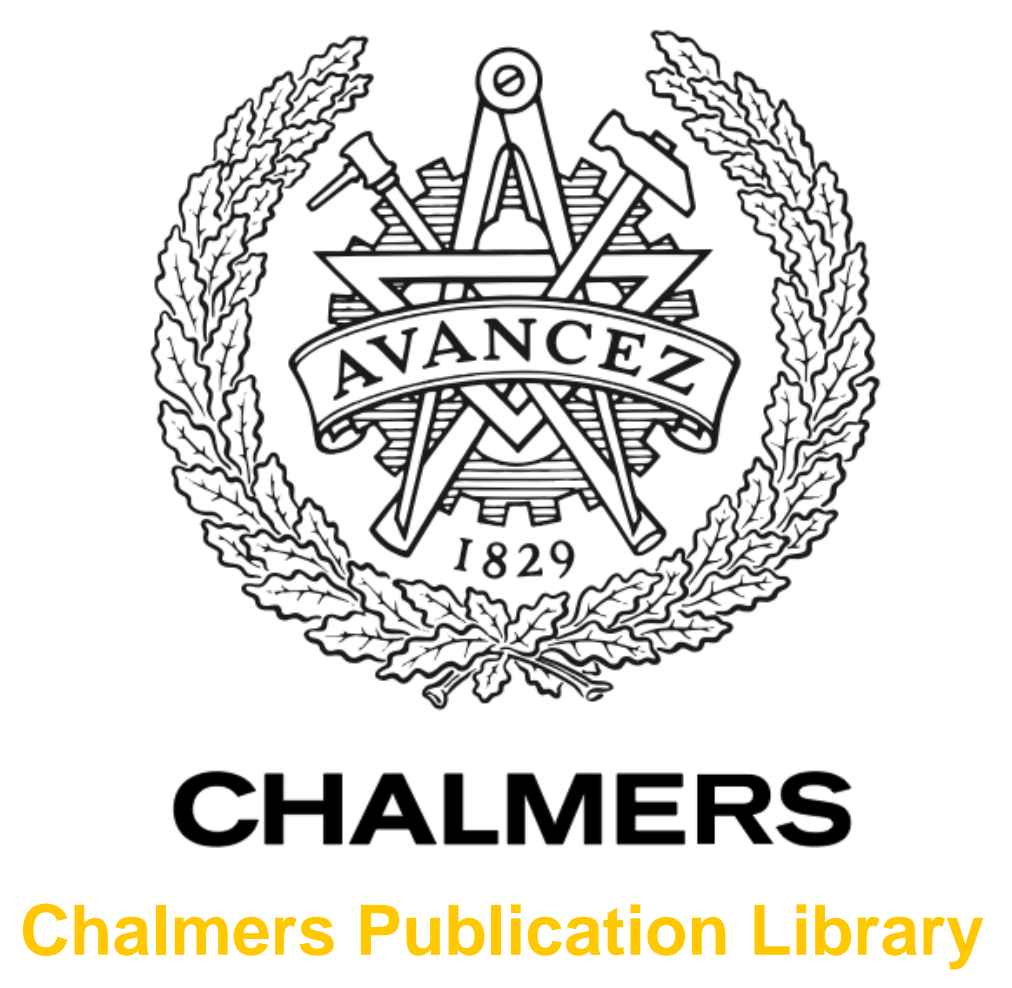

\title{
Behaviour and stability of the two-fluid model for fine-scale simulations of bubbly flow in nuclear reactors
}

This document has been downloaded from Chalmers Publication Library $(\mathrm{CPL})$. It is the author's version of a work that was accepted for publication in:

International journal of Chemical Reactor Engineering (ISSN: 1542-6580)

Citation for the published paper:

Ström, H. ; Sasic, S. ; Jareteg, K. et al. (2015) "Behaviour and stability of the two-fluid model for fine-scale simulations of bubbly flow in nuclear reactors". International journal of Chemical Reactor Engineering

Downloaded from: http://publications.lib.chalmers.se/publication/223513

Notice: Changes introduced as a result of publishing processes such as copy-editing and formatting may not be reflected in this document. For a definitive version of this work, please refer to the published source. Please note that access to the published version might require a subscription. 
Open Access

Henrik Ström*, Srdjan Sasic, Klas Jareteg and Christophe Demazière

\section{Behaviour and Stability of the Two-Fluid Model for Fine-Scale Simulations of Bubbly Flow in Nuclear Reactors}

\section{DOI 10.1515/ijcre-2014-0171}

\begin{abstract}
In the present work, we formulate a simplistic two-fluid model for bubbly steam-water flow existing between fuel pins in nuclear fuel assemblies. Numerical simulations are performed in periodic 2D domains of varying sizes. The appearance of a non-uniform volume fraction field in the form of meso-scales is investigated and shown to be varying with the bubble loading and the domain size, as well as with the numerical algorithm employed. These findings highlight the difficulties involved in interpreting the occurrence of instabilities in two-fluid simulations of gas-liquid flows, where physical and unphysical instabilities are prone to be confounded. The results obtained in this work therefore contribute to a rigorous foundation in on-going efforts to derive a consistent meso-scale formulation of the traditional two-fluid model for multiphase flows in nuclear reactors.
\end{abstract}

Keywords: two-phase flow, bubbly flow, gas-liquid flow, nuclear reactors

\section{Introduction}

The modeling of the current fleet of nuclear reactors traditionally relies on macroscopic approaches modeling different fields of physics, namely neutron transport, fluid dynamics, and heat transfer, among others. The reason behind the choice of macroscopic models lies in the fact that the verification and validation of models being used by the nuclear industry is a lengthy and

*Corresponding author: Henrik Ström, Division of Fluid Dynamics, Department of Applied Mechanics, Chalmers University of Technology, SE-412 96 Göteborg, Sweden, E-mail: henrik.strom@chalmers.se Srdjan Sasic, Division of Fluid Dynamics, Department of Applied Mechanics, Chalmers University of Technology, SE-412 96 Göteborg, Sweden

Klas Jareteg, Christophe Demazière, Division of Nuclear Engineering, Department of Applied Physics, Chalmers University of Technology, SE-412 96 Göteborg, Sweden expensive process that involves many actors, such as research institutes, code manufacturers, plant and fuel manufacturers, and safety regulatory bodies. The industry is thus heavily relying on capitalization, i.e. on the continuous improvement of codes and models, rather than on developing of entirely new modeling approaches. In this respect, the advancement of computer resources in the 1970s resulted in the start-up of large developmental projects in the simulation of nuclear reactors. Due to the size of such systems though, and because of the rather limited computing power available at that time, macroscopic models represented the only possible modeling approach. In the area of two-phase flow, this resulted in the development of the two-fluid model at a macroscopic level, where the highfrequency and small scale filtered phenomena were artificially introduced via the use of experimentally-derived closure relationships.

The present work investigates the performance of the two-fluid model on smaller scales than what is typically used in the nuclear industry today. In the investigations reported hereafter, we formulate a simplistic two-fluid model for bubbly steam-water flow. Numerical simulations are performed in periodic 2D domains of varying sizes, investigating the spontaneous emergence of nonuniform bubble volume fraction fields in the form of meso-scales. The results obtained in this work contribute to a rigorous foundation in on-going efforts to derive a formulation of the traditional two-fluid model for multiphase flows in nuclear reactors that takes the effects of meso-scales into account.

It is well known, particularly from the literature on gas-solid flows, that solutions to the conventional twofluid model exhibit the emergence of meso-scale structures at sufficiently high volume fractions of the dispersed phase. These structures can be seen as non-uniformities in the obtained volume fraction fields and thus represent structures whose characteristic sizes are of the order of several (more than ten) dispersed phase diameters. Falling in between the micro-scales (determined by the size of the dispersed phase) and the macro-scales 
(determined by the size of the bounding geometry), these structures are termed meso-scale structures. The formation of these structures is due to clustering of the dispersed phase and takes place because of inherent instabilities of the system. Meso-scale structures may therefore manifest also under uniform flow conditions in unbounded domains. The occurrence of meso-scale structures in numerical simulations of two-phase flow using the twofluid model was first investigated by Agrawal et al. (2001) and has since then received great interest. Investigations of instabilities and filtering approaches for taking the effects of unresolved meso-scale structures into account in coarse-grid two-phase simulations has also been investigated in great detail, mostly for gas-solid flows (Agrawal et al. 2001; Zhang and VanderHeyden 2001; Benyahia and Sundaresan 2012; Wang, van der Hoef, and Kuipers 2009) but also more recently for gas-liquid flows (Yang et al. 2011). It has been shown that the appearance of instabilities related to clustering and voidage formation in twophase systems requires neither macroscopic shear nor the presence of boundaries (Agrawal et al. 2001). In fact, it is sufficient that there is an interaction between the inertia of the dispersed phase, gravity and the interphase drag (Agrawal et al. 2001).

The motion and stability of bubbly suspensions have been studied on single bubbles in periodic 2D- and 3Ddomains (i.e. representing an infinite regular array of rising bubbles) (Sankaranarayanan and Sundaresan 2002; Sankaranarayanan et al. 2002), and it is known that the uniform bubbling state loses stability at some critical bubble volume fraction (Joshi et al. 2001; Mudde, Harteveld, and van den Akker 2009). Freely evolving swarms of near-spherical two-dimensional bubbles were also shown to produce meso-scale structures, although they rise faster than a regular array of bubbles at the same volume fraction (Esmaeeli and Tryggvason 1998).

Several different scenarios have been proposed for the underlying physical mechanisms responsible for a transition from a homogeneous to a heterogeneous bubbly flow. Sankaranarayanan and Sundaresan (2002) found that the hindered motion of the dispersed phase gives rise to vertically travelling waves (just as in gassolid flows) whereas cooperative motion of the dispersed phase tends to create columnar structures. They also showed that a lift force is not necessary to trigger an instability. On the other hand, Lucas, Prasser, and Manera (2005) and Lucas et al. (2006) concluded that the lift force is of utmost importance, and that a positive sign of the lift force coefficient practically acts as a stability criterion. Finally, Monahan and Fox (2007) attributed the loss of stability to yet another mechanism - that bubble wakes are suppressed as the gas holdup increases, which triggers the instabilities due to a decreased effective viscosity. Even though the experiments of Mudde, Harteveld, and van den Akker (2009) show that uniform bubbly flows are indeed unstable, theoretical works seem to predict lower critical gas fractions for the transition to unstable behaviour. We find this observation very interesting, as we believe that it hints at some of the difficulties in interpreting the occurrence of instabilities in two-fluid model predictions of gasliquid flows.

In addition to the physical reasons for the unstable behaviours observed in gas-liquid two-phase flow, there are namely also well-documented mathematical difficulties associated with the two-fluid model as such. The most commonly used version of the two-fluid model for gas-liquid flows is based on an assumption of pressure equilibrium between the phases, which leads to a non-hyperbolic illposed model in the inviscid limit (Dinh, Nourgaliev, and Theofanous 2003; Yström 2001; Prosperetti and Satrape 1990; Ransom and Hicks 1984, 1988). Although it has been argued that the ill-posedness arises when local discontinuities (i.e. interfaces) are homogenized by an averaging procedure, there is still no general consensus on whether the non-hyperbolicity of the equation set reflects the presence of physical instabilities or whether instabilities are artificially augmented by an inadequate mathematical representation (Dinh, Nourgaliev, and Theofanous 2003; Prosperetti and Satrape 1990). In practice, the appearance of instabilities in numerical solutions to the two-fluid model is often suppressed by the use of excessively dissipative numerical schemes or by regularization of the physical model (Prosperetti and Satrape 1990; Pokharna, Mori, and Ransom 1997). Unfortunately, little is known about the effect of such measures on the simulation of large-scale flow oscillations over long times (Prosperetti and Satrape 1990; Pokharna, Mori, and Ransom 1997; Stewart 1986). Understanding the uncertainties inherent in these types of simulations is absolutely critical in an effort to use numerical results obtained with a two-fluid model at fine resolution to derive appropriate relations for the effects of unresolved meso-scale structures at a coarser resolution. Most notably, there is a significant risk that solutions of an ill-posed model might suffer from excessive numerical diffusion and/or exhibit unphysical instabilities (Dinh, Nourgaliev, and Theofanous 2003), which could inadvertently be transferred along to the meso-scale closures.

The aim of the current work is to investigate the performance of the two-fluid model for nuclear reactor applications. More specifically, the aim is to assess the dependence of the behaviour and stability of the two- 
fluid model on the specifications of the computational setup and the case properties in a vein similar to that of previous studies related to gas-solid flows (Agrawal et al. 2001; Zhang and VanderHeyden 2001; Benyahia and Sundaresan 2012; Wang, van der Hoef, and Kuipers 2009). The results obtained in this work may therefore form a rigorous foundation in on-going efforts to devise a meso-scale formulation of the traditional two-fluid model for multiphase flows in nuclear reactors, as the two-fluid model used here will be subsequently extended to also allow descriptions of other forms of momentum exchange, as well as heat and mass transfer.

\section{Modelling}

This work investigates the occurrence of instabilities, fluctuations and deviations from the uniform state in two-fluid model simulations of bubbly flow in nuclear reactors. Consequently, the two-fluid model employed must be made simple enough to enable the direct observation of the effects of each feature added to the model. It is therefore assumed that the flow of interest can be represented by spherical, rigid bubbles occupying a certain volume fraction in a continuous liquid. The momentum exchange between the two phases is assumed to be dominated by the drag force. The flow is isothermal and there is no mass transfer between the phases. There is no coalescence or breakup of bubbles. These assumptions are not adequate for a real nuclear reactor, but are deemed necessary to make the formulation of a two-fluid model relevant for the steam-water flow that is stripped of all complexities except for those inherent in the two-fluid model.

\subsection{Simplistic two-fluid model}

The two-fluid model formulated for a continuous liquid phase and a mono-sized dispersed bubbly phase, under the set of restrictive assumptions noted previously, is presented in the following. The continuity equation for the dispersed phase becomes:

$$
\frac{\partial}{\partial t}\left(\alpha_{b} \rho_{b}\right)+\nabla \cdot\left(\alpha_{b} \rho_{b} \boldsymbol{u}_{b}\right)=0
$$

The continuous phase volume fraction field is obtained from the condition that the sum of the volume fractions everywhere must be equal to unity:

$$
\alpha_{l}=1-\alpha_{b}
$$

In this notation, $l$ represents the liquid phase and $b$ the bubbly phase.

The momentum balance equations become:

$$
\begin{aligned}
& \frac{\partial}{\partial t}\left(\alpha_{k} \rho_{k} \boldsymbol{u}_{\boldsymbol{k}}\right)+\nabla \cdot\left(\alpha_{k} \rho_{k} \boldsymbol{u}_{k} \boldsymbol{u}_{k}\right)= \\
& -\alpha_{k} \nabla p+\nabla \cdot\left[\alpha_{k} \mu_{k}\left(\nabla \boldsymbol{u}_{k}+\nabla \boldsymbol{u}_{k}{ }^{T}\right)\right]+\alpha_{k} \rho_{k} \boldsymbol{g}+K\left(\boldsymbol{u}_{q}-\boldsymbol{u}_{k}\right)
\end{aligned}
$$

Here, $k$ is either $l$ or $b$, and $q$ is the other phase (not currently represented by $k$ ). The pressure $p$ is shared by both phases, and the viscosity used is the sum of the turbulent viscosity (when a turbulence model is used) and the molecular viscosity of the continuous phase.

Two things should be emphasized in relation to (eq. (3)): Firstly, there is no discrete phase pressure and thus no repulsive term that prevents overpacking of the discrete entities. This choice is in line with the current ambition to perform a transparent investigation with as few complications as possible. At relatively low discrete phase volume fractions, such as in this work, overpacking is generally not an issue and this approach can be justified $a$ posteriori by investigations of the obtained volume fraction fields. At higher volume fractions, a dispersed phase pressure would be needed to prevent overpacking, but such models are known to be sensitive to the choices of model parameters (Benyahia and Sundaresan 2012). In this work, the isotropic contribution to the discrete phase stresses thus come from the shared pressure field. Secondly, the deviatoric contribution to the discrete phase stresses is here obtained from the product of the continuous phase viscosity with the discrete phase velocity gradients. In similar previous studies, other approaches have been suggested for the treatment of these terms, such as neglecting them altogether (Benyahia and Sundaresan 2012) or to assume them to be identical to the continuous phase deviatoric stresses (Zhang and VanderHeyden 2001). As the effect of the deviatoric contribution to the discrete phase stresses is related to the growth rate of disturbances in the flow field (the discrete phase viscosity determines the length scale of the dominant instability), but not to the existence of disturbances as such (Anderson, Sundaresan, and Jackson 1995), the treatment of these terms is deemed acceptable for the purpose of the current work. Furthermore, it has been shown for gas-solid flows that the meso-scale stresses dominate over the molecular stresses (Agrawal et al. 2001).

The momentum exchange coefficient $K$ is obtained using the following relation:

$$
K=\alpha_{l} \alpha_{b} \frac{18 \mu_{l}}{d_{b}^{2}} \frac{C_{D} R e_{p}}{24}
$$


Here, $C_{D}$ is evaluated using the Morsi and Alexander drag law (Morsi and Alexander 1972), which is applicable for spherical particles over a wide range of particle Reynolds numbers (from the Stokes flow regime up to above 10,000).

The two-fluid model used here is somewhat simpler than a conventional two-fluid model for nuclear applications. We therefore stress that there has been no loss of generality from the simplifications involved with respect to the occurrence of instabilities. The origin of the mathematical instabilities lies in the one-pressure formulation and the energy equations, if included, do not affect the hyperbolicity of the equation set (Ransom and Hicks 1984, 1988). Furthermore, the model is comprehensive enough to capture at least some of the possible physical instabilities known to exist in gas-liquid flows (Agrawal et al. 2001; Sankaranarayanan and Sundaresan 2002). In addition, the main emphasis in the current work is not on wall-bounded systems, where velocity gradients develop and may affect the stability of a uniformly bubbling suspension via the bubble lift forces (Sankaranarayanan and Sundaresan 2002; Lucas, Prasser, and Manera 2005; Lucas et al. 2006). Instead, we aim to study whether loss of stability is possible in a periodic domain.

The two-fluid model derived in this way and applied to a periodic domain is well-posed locally in time if the initial data are smooth, but is known to possess a medium to high wavenumber instability. In practice, a smooth solution to such a two-fluid model is therefore exponentially unstable. Linearization around an assumed smooth solution, followed by a freezing of coefficients, yields that the exponential growth rate of the instabilities is (to the first order) (Gudmundsson 2005):

$$
r=\frac{\left(\left|\boldsymbol{u}_{r}\right|\right)^{2} \alpha_{b} \alpha_{l}\left(\rho_{l} \mu_{b}^{2} \alpha_{l}+\rho_{b} \mu_{l}^{2} \alpha_{b}\right)}{\left(\mu_{l} \alpha_{b}+\mu_{b} \alpha_{l}\right)^{3}}
$$

Although it is still unclear how well this result transfers to the original non-linear problem (Gudmundsson 2005; Keyfitz 2001), it is deemed valuable in the assessment of the growth rate observed in our simulations. It has also been shown that, even though there is an exponential instability, the solutions can still be bounded if they become highly oscillatory or if they form shock-like structures (Gudmundsson 2005; Keyfitz 2001; Kreiss and Yström 2002). In practice, the numerical results obtained will be influenced also by the algorithms and discretization schemes used in the solution procedure (Coquel et al. 1997). It is the purpose of this work to investigate the sensitivity of the obtained solutions to both the physics (as specified by the model itself and its initial and boundary conditions) and to the numerics.

\subsection{Standard k- $\varepsilon$ model}

The bubbly flow inside a nuclear reactor is highly turbulent, which typically motivates the inclusion of a turbulence model in the computational framework (Bestion 2012). On the other hand, if large-scale vortical structures are expected to be a more significant source of velocity fluctuations than bubble- or shear-induced turbulence (as should indeed be expected for a fully periodic bubbly flow at low to moderate bubble loading), a turbulence model is generally not needed (Ojima et al. 2014). In this work, we compare simulations using a turbulence model (Section 3.5) with simulations where no turbulence model is employed (Sections 3.1-3.4). In line with the aim to keep the current investigation transparent and simplistic, the standard k- $\varepsilon$ model (Launder and Spalding 1972) is chosen. Note that the model is applied to the mixture and not for the individual phases. However, it should be stressed here that due to the large density difference between the phases, the modeled turbulence will be most significantly affected by the liquid phase. Even though turbulence modeling in dense, dispersed two-phase flows is a highly complex issue, this approach is deemed appropriate given the aim and scope of the current work.

\subsection{Computational cases}

The pressure-velocity coupling is handled via the phasecoupled SIMPLE algorithm (Vasquez and Ivanov 2000). In the discretization of the convective terms of all balance equations, the third-order accurate, bounded QUICK scheme is used with structured hexahedral meshes. The diffusion terms are discretized using a second-order accurate central-differencing scheme.

The geometry chosen is a fully periodic 2D system similar to that used by Benyahia and Sundaresan (2012). The domain height is four times its width $(0.1 \times 0.4 \mathrm{~m})$, and gravity acts downwards in the vertical direction. The weight of the carrier and the dispersed phase in the domain is balanced exactly by a prescribed pressure drop in the vertical direction. This system is not typical of the flow channels in nuclear cores, but designed to represent a situation where the flow is unbounded. The material properties are assumed constant. The spatial discretization, unless otherwise noted, is the finest one used by Benyahia and Sundaresan (2012), i.e. 64 by 256 . 
The trends reported in this work were confirmed to be mesh-independent with this resolution.

The choice to use a 2D domain is based on the need to reduce the computational cost when performing many simulations over long times. It should therefore be stressed that physical meso-scale structures, as defined here, arise as the continuous phase will bypass clusters of the dispersed phase more easily than it will flow through a completely homogenized system. This effect therefore appears in both two- and three-dimensional numerical simulations, albeit with quantitative differences (Agrawal et al. 2001). Furthermore, instabilities of unphysical origin are known to manifest already in 1D simulations (Pokharna, Mori, and Ransom 1997).

Since the purpose of the current work is to investigate the possible onset of instabilities inherent in the two-fluid formulation applied to typical nuclear reactor cases, we are not primarily interested in whether such instabilities eventually develop at all, but rather whether they develop over length and time scales relevant to the thermo-hydraulics of nuclear reactors. Taking $4 \mathrm{~m}$ as a representative height of a nuclear reactor (Demazière 2013) and $2 \mathrm{~m} / \mathrm{s}$ as a representative liquid velocity (Anglart et al. 1997; Ustinenko et al. 2008), we obtain a macroscopic time scale of $2 \mathrm{sec}$. Hence, in theory it should be sufficient to study the system for a period of $2 \mathrm{sec}$. On the other hand, all simulations are started at time zero with a uniform volume fraction field, and it is likely that the time needed for instabilities to manifest will be the longest for such initial conditions. In order not to neglect instabilities that would develop within a short time frame only if the initial conditions are favorable, we therefore choose to study a longer time period of $20 \mathrm{sec}$. This time period corresponds to more than 100 passages through the periodic computational domain in the streamwise direction for the mean flow.

As a means to quantify the magnitude of the mesoscale structures, we define a global, time-resolved uniformity index $\Phi(t)$ such that:

$$
\Phi(t)=\frac{\alpha_{q, \max }(t)-\alpha_{q, \min }(t)}{\alpha_{q, \text { avg }}}
$$

where $\alpha_{q, \max }$ is the current maximum volume fraction of phase $q$ in the solution domain at time $t, \alpha_{q, \min }$ is the corresponding current minimum volume fraction and $\alpha_{q, a v g}$ is the domain-average value of the volume fraction. With this definition, a value of $\Phi$ equal to zero corresponds to a uniform volume fraction field and complete phase separation would correspond to $\Phi=1 / \alpha_{q, a v g}$. The occurrences of non-zero values of $\Phi$ with time are indicative of the appearance of a non-uniform volume fraction field.

\section{Results and discussion}

\subsection{Case \#1 - Gas-solids case}

We first investigate the same gas-solids flow as the one that was simulated by Benyahia and Sundaresan (2012). The case specification is presented in Table 1 . The simulation is started with a uniform volume fraction field of the dispersed phase and advanced in time with a time step of $10^{-4} \mathrm{~s}$. The simulation results for the volume fraction field are shown in Figure 1. Using the results from Benyahia and Sundaresan (2012) as a reference solution, it is verified that our numerical setup can reproduce the qualitative behavior of this gas-solids flow, including the appearance of meso-scale structures and fluctuations in the slip velocity, and we use this setup as a basis for going towards bubbly flow relevant to nuclear reactors.

Table 1: Gas-solids case.

\begin{tabular}{ll}
\hline System size (width $\times$ height), $\mathrm{m}$ & $0.1 \times 0.4$ \\
Fluid density, $\rho_{f} \mathrm{~kg} \mathrm{~m}^{-3}$ & 1.3 \\
Fluid viscosity, $\mu_{f} \mathrm{~Pa} \mathrm{~s}$ & $1.8 \times 10^{-5}$ \\
Particle density, $\rho_{p} \mathrm{~kg} \mathrm{~m}^{-3}$ & 1,500 \\
Particle diameter, $d_{p} \mu \mathrm{m}$ & 75 \\
Average particle volume fraction & 0.05 \\
\hline
\end{tabular}

\subsection{Case \#2-gas-liquid case 1}

As a first test for a gas-liquid system, we adjust Case \#1 so that the terminal velocity of a bubble is similar to that of the solid particles. Under the simplified assumption of Stokes flow around the dispersed phase, the new bubble size can be found from:

$$
d_{b}=d_{p} \sqrt{\frac{\mu_{l}}{\mu_{g}} \frac{\left|\rho_{s}-\rho_{g}\right|}{\left|\rho_{b}-\rho_{l}\right|}}
$$

We then rerun Case \#1 with the material properties of both phases representative of a gas-liquid flow (cf. Table 2). The properties are chosen to reflect relevant orders of magnitude, and could represent either an airwater system or a steam-water system (with the steam being either saturated or superheated). All other parameters are kept the same.

The resulting bubble diameter for this case is $0.68 \mathrm{~mm}$, which is a relevant bubble size for the onset of bubble creation at sub-cooled boiling conditions (Anglart et al. 

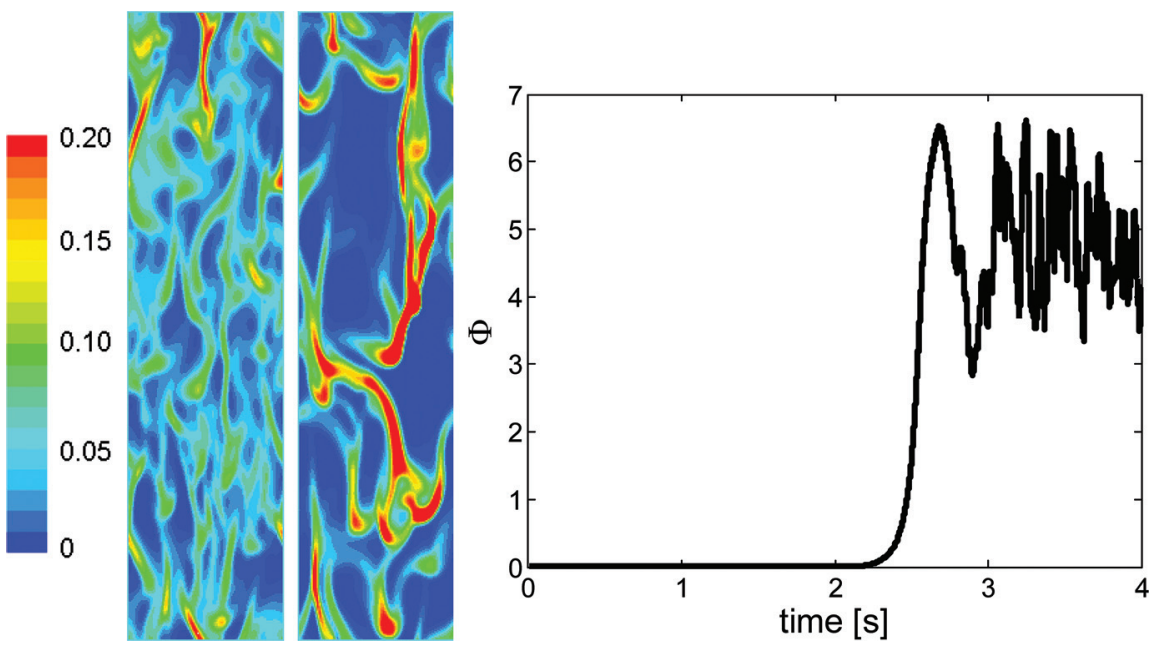

Figure 1: Snapshots of discrete phase volume fraction at $t=2.5 \mathrm{~s}$ (left) and $3.5 \mathrm{~s}$ (middle). Blue and red indicate dilute and dense (volume fraction of 0.2 and higher) flow regions. To the right: Time-resolved uniformity index for Case \#1.
Table 2: Gas-liquid case.

\begin{tabular}{ll}
\hline Fluid density, $\rho_{f} \mathrm{~kg} \mathrm{~m}^{-3}$ & 1,000 \\
Fluid viscosity, $\mu_{f} \mathrm{~Pa} \mathrm{~s}$ & $1 \times 10^{-3}$ \\
Particle density, $\rho_{p} \mathrm{~kg} \mathrm{~m}^{-3}$ & 1 \\
\hline
\end{tabular}

1997). The behavior of the time-resolved uniformity index is shown in Figure 2. There are clear non-zero values after a few seconds of real time. A visual inspection of the volume fraction field (Figure 3) reveals meso-scale structures in the form of very thin vertically stretched zones of bubble-rich and bubble-lean areas.
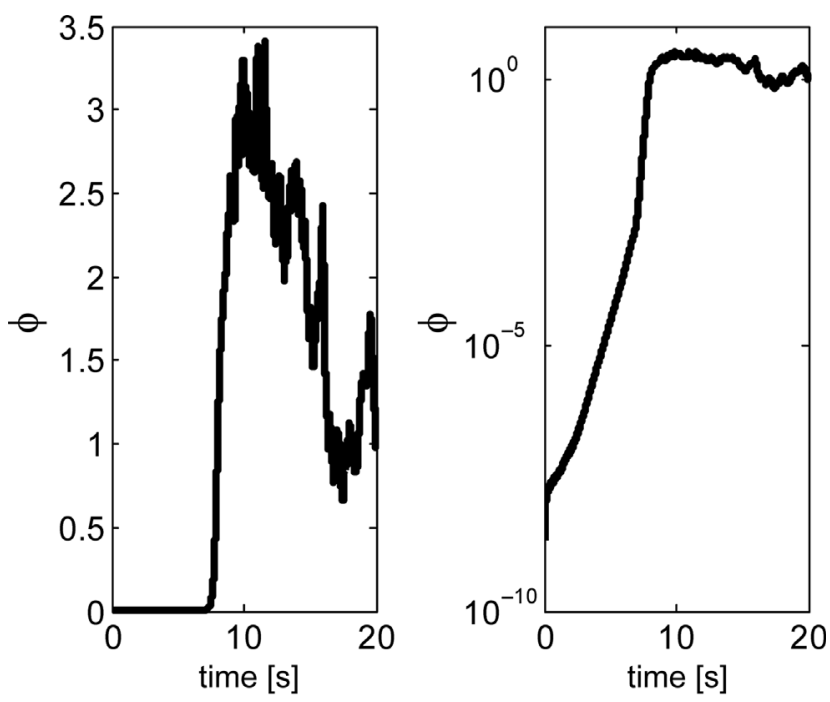

Figure 2: Time-resolved uniformity index for Case \#2. To the left: linear scale, to the right: semi-log scale.

Soon after the emergence of the "striped" structures in Figure 3, the time-resolved uniformity index starts to
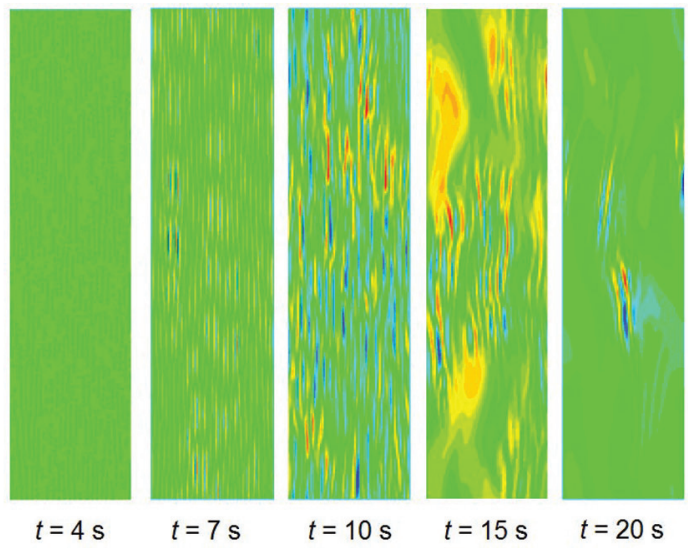

$t=15 \mathrm{~s}$

$t=20 \mathrm{~s}$
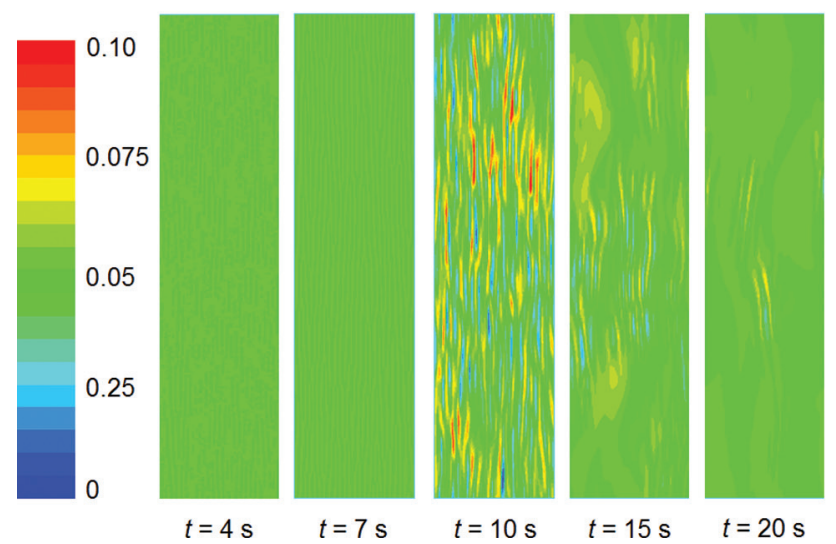

Figure 3: Snapshot of the discrete phase volume fractions in Case \#2. Top: scaled by the maximum and minimum values observed in each snapshot. Bottom: scaled by the maximum and minimum values observed throughout the simulation (colour legend to the left). Streaks of high (red) and low (blue) volume fraction emerge slowly as a "striped" pattern and can be clearly discerned after $10 \mathrm{sec}$.

decrease slowly and the meso-scale structures become less sharp and develop a smoother, somewhat undulating character. These phenomena are attributed to the 
exponential growth of small initial disturbances (in the uncertainty pertaining to the numerical precision). The solution eventually goes through a maximally unstable state, where gradients become so large that they will start to become smoothed out again by diffusive mechanisms. These diffusive mechanisms originate both from the physical model and from the numerical procedure. In the present model, it is primarily the viscous stresses that tend to stabilize disturbances (Arai 1980), but also the momentum exchange between the phases could play a similar role (Stewart 1979). There are several steps in the numerical solution procedure that influence stability, most notably the discretization of the convective terms in (eqs (1) and (3)) (Toumi 1996 and Prosperetti 2003). After the peak in the instability, the solution remains non-homogeneous throughout the rest of the simulation.

These observations are consistent with the previous literature investigations of the stability of the two-fluid model: the current formulation is unstable, but its solutions are still bounded. It is also interesting to note that the (material and case-specific) properties of this bubbly flow are very similar to one of the examples investigated by Sankaranarayanan and Sundaresan (2002). For this system, they found that the bubbles remain nearly spherical and the bubbly flow loses stability due to a growth of vertically traveling wavefronts having no horizontal structure. This mode is associated with the inertia of relative motion between the two phases and is thus the same as the dominant instability mode in typical gas-solid flows. The lift force, which is not taken into account in the present work, plays an important role in the loss of stability through a different mode and is thus not required for these instabilities to manifest (Sankaranarayanan and Sundaresan 2002). On the contrary, the addition of a lift force or a discretephase pressure to the model would act so as to stabilize the system under these conditions (Sankaranarayanan and Sundaresan 2002; Gudmundsson 2005; Coquel et al. 1997).

The effect of the numerical procedure to obtain the solution on the behavior and the stability of the solution itself is illustrated in Figure 4, where the results are compared for two simulations that differ only in the choice of either a lower-order scheme (First Order Upwind) or a higher-order scheme (QUICK) in the discretization of the convective terms in all balance equations. The First Order Upwind scheme is known to be robust but to give rise to false (numerical) diffusion, which should generally be expected to increase the stability and delay the transition to the unstable state on the same mesh. These are also the inferences that indeed can be drawn from Figure 4 . Additionally, it is observed that neither scheme produces a growth rate as large as the theoretical one predicted by

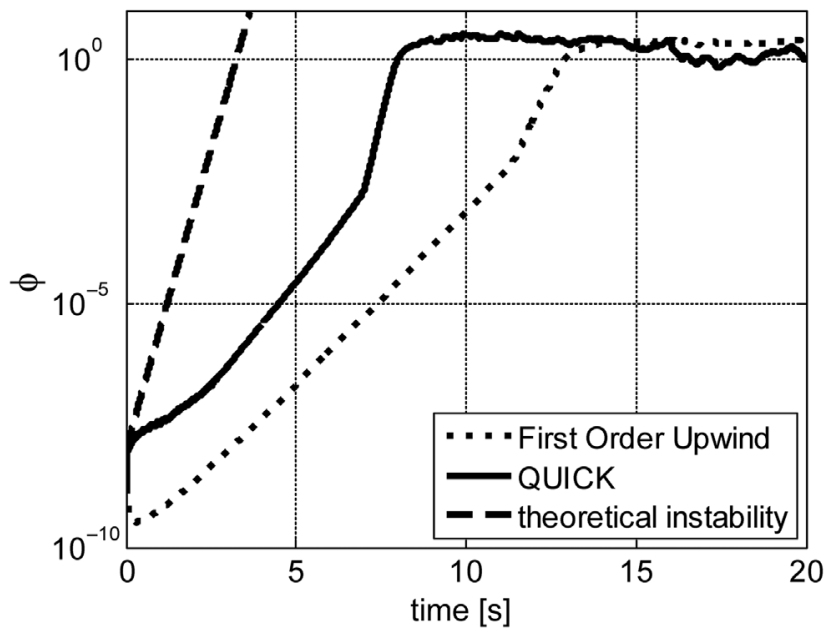

Figure 4: Time-resolved uniformity index for Case \#2: the effect of using a lower-order (First Order Upwind) or a higher-order (QUICK) discretization scheme for the convective terms in (eqs (1) and (3)). The theoretical growth rate for the instabilities obtained from (eq. (5)) is also plotted for comparison.

(eq. (5)). It is also concluded that the effect of the choice of the numerical solution procedure on the behavior of the system is significant, and it should thus be emphasized that the exact time-history obtained (as shown in Figure 3) is unlikely to reflect a true physical behavior of the system, but should be interpreted rather as a display of the unstable character of a bubbly two-phase flow and the complex interplay between the design of the mathematical model and the choice of numerical algorithms dedicated to solving it.

Ransom and Hicks $(1984,1988)$ use the term "unphysical instabilities" to differentiate unbounded instabilities from bounded instabilities (which they refer to as "physical instabilities"), and they argue that mathematical models of physical instabilities (e.g. interface instabilities in two-phase flows) will start to exhibit unphysical behavior when the physics included in the model is insufficient (Ransom and Hicks 1984, 1988). They propose that instabilities will result from the inadequate pressure equilibrium assumption inherent in the single-pressure twofluid model, but that these instabilities may remain bounded because of the combined effects of, for example, viscous stresses and numerical diffusion. These speculations agree with our observations. As the simulations are advanced in time, the miniscule numerical round-off errors in the predicted pressure, velocity and volume fraction fields tend to grow with time throughout the domain.

Given the fact that the current formulation of the twofluid model for a bubbly gas-liquid flow exhibits these characteristics, we will now investigate the sensitivity of this behavior to changes in the case specification 
(e.g. changes to the bubble loading and the domain size) and to the inclusion of a turbulence model.

\subsection{Case \#3 - Gas-liquid case 2}

It is known that a uniformly bubbling suspension loses stability at bubble loadings of a few percent or more, and that the suggested critical bubble loadings span a wide range (cf. Sankaranarayanan and Sundaresan (2002), Joshi et al. (2001), Mudde, Harteveld and van den Akker (2009)). However, the simplistic formulation of the two-fluid model that is under investigation here is known to be unstable for all non-zero bubble loadings (Gudmundsson 2005). Numerical simulations are performed to investigate the behavior of the volume fraction fields predicted by this two-fluid model for three different average bubble loadings: 1, 5 and 10\%. The results are shown in Figure 5. It is clear that the growth rate of the initial instabilities is a function of the bubble loading, and that the growth rate increases with increasing loading. The non-uniformities in the case with $1 \%$ bubble loading are still extremely small after $20 \mathrm{sec}$, but a growth is still clearly there. The behavior at 5 and $10 \%$ bubble loading are qualitatively similar, with the difference that stability is lost earlier with the higher bubble loading.

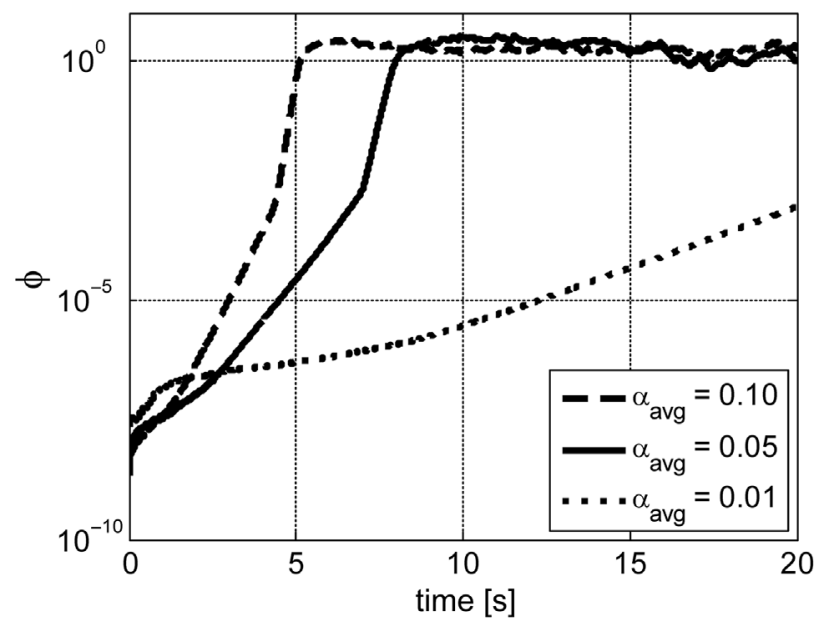

Figure 5: Time-resolved uniformity index for Case \#3.

\subsection{Case \#4 - Nuclear reactor case 1}

The bubbly flow in a nuclear reactor occurs in between the fuel rods and is therefore geometrically restricted in the horizontal direction. Representative values of the distance between the centers of the fuel rods are in the range 1-3 cm, with the distance between the outer radii of two consecutive fuel pins being approximately $3-8 \mathrm{~mm}$
(Anglart et al. 1997; Ustinenko et al. 2008). In other words, the characteristic length of the bounding geometry in a nuclear reactor case is approximately an order of magnitude smaller than in the previous computational cases. At the same time, the variation in the typical bubble size can be very significant in the vertical direction. The initial creation of bubbles at sub-cooled boiling conditions, however, results in small, spherical bubbles of 0.15-1.5 mm in diameter (Anglart et al. 1997), but bubble diameters up to $5 \mathrm{~mm}$ are relevant (Ustinenko et al. 2008). The resulting ratio between the discrete phase diameter and the length scale characterizing the bounding geometry is thus of the order of $0.015-0.625$ in a nuclear reactor, whereas it was 0.0068 in the previous computational case. In other words, for the investigations to be fully relevant to nuclear reactor applications, it is necessary to also study higher values of $d_{p} / L$. Here, we choose to scale the computational domain so that it becomes $1 \mathrm{~cm}$ wide (maintaining the aspect ratio) and to maintain a bubble diameter of $0.68 \mathrm{~mm}$, yielding a value of $d_{p} / L$ equal to 0.068 . This value is in the correct range and represents a significant increase from the previous cases. Finally, we also change the boundary conditions on the vertical sides from periodic to free-slip walls. The presence of a wall introduces the sought-after geometrical limitation on the meso-scale structures, and the free-slip boundary condition allows us to probe the influence from this restriction without imposing sharp gradients in the velocity field that are likely to have an additional influence of their own on the instabilities.

The time-resolved non-uniformity index obtained with a $1 \mathrm{~cm}$ wide, horizontally bounded geometry with free-slip walls is displayed in Figure 6. The instabilities are there but the non-uniformity index remains lower

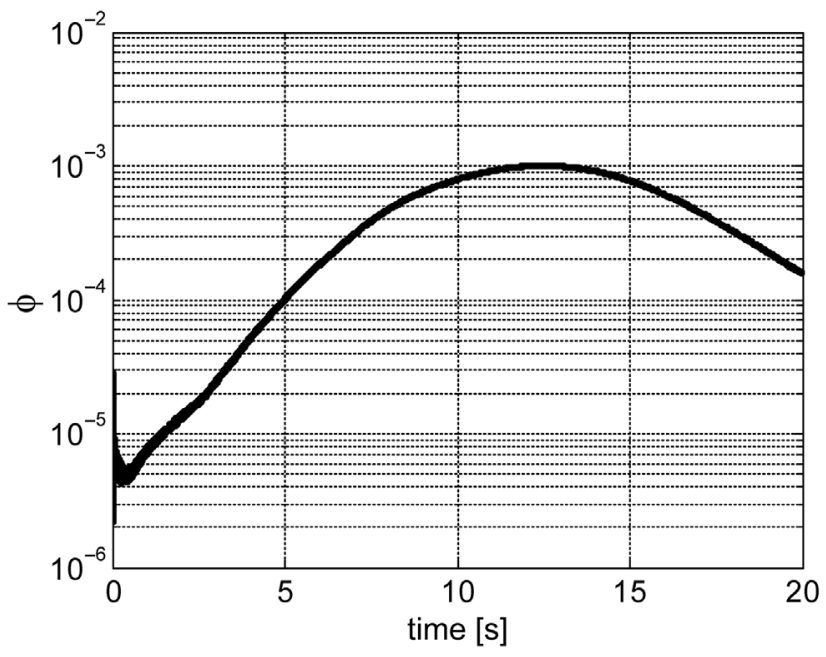

Figure 6: Time-resolved uniformity indices for Case \#4: a small $(1 \mathrm{~cm})$ domain with free-slip walls. 
than $10^{-3}$ for the first $20 \mathrm{sec}$ for this case. The effects of imposing a no-slip boundary condition are shown in Figure 7 for the original $10 \mathrm{~cm}$ wide domain. The large gradients in the velocity field that result from the no-slip boundary condition trigger the instabilities much faster than for the fully periodic case. Here, meso-scale structures appear already within the first $3 \mathrm{sec}$. The trend with respect to the domain size is as expected: the instabilities are less pronounced in a smaller $(1 \mathrm{~cm})$ domain, but their magnitude are still larger when employing no-slip boundary conditions than with free slip.

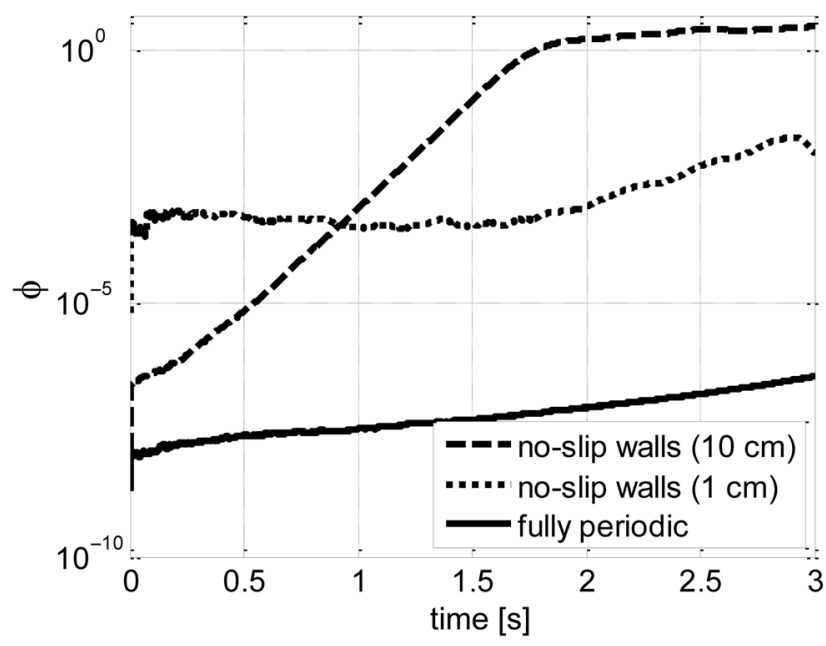

Figure 7: Time-resolved uniformity indices for Case \#4: the effect of no-slip walls.

\subsection{Case \#5 - Nuclear reactor case 2}

As described previously, the two-fluid model is typically used together with a Reynolds-Averaged Navier-Stokes (RANS)-based turbulence model in the simulations of nuclear reactors. The most important effect of adding a RANS model to these computations is the introduced change in the effective viscosity. Consequently, we revisit the previous nuclear reactor cases with the addition of the Standard $\mathrm{k}-\varepsilon$ turbulence model.

The Standard $k-\varepsilon$ model predicts energy-containing turbulent eddies of approximately $1.8 \mathrm{~cm}$ in the bounded $10 \mathrm{~cm}$ domain. The ratio of the turbulent to the dynamic viscosity of the liquid becomes of the order of several hundreds, implying that the effective viscosity is significantly increased, as expected. In comparison, large eddy simulations of the turbulent liquid (single-phase) flow in a three-dimensional mesh of identical resolution with the dynamic Smagorinsky subgrid model yields turbulent viscosities of the same order of magnitude as the molecular viscosity or lower. These large eddy simulations hence suggest that the mesh resolution is almost sufficient for a direct numerical simulation of the turbulence.

The effect on the meso-scale structures from the addition of the Standard k- $\varepsilon$ model is to effectively dampen out all fluctuations, and the non-uniformity indices (not shown) remain very small throughout the simulations. This observation is not so strange after all, since it has been known for decades that the addition of an "artificial viscosity" in the numerical algorithm employed to solve the two-fluid model helps dampen out highfrequency oscillations and therefore assists in achieving numerical stability (Ransom and Hicks 1984; Ishii and Mishima 1984).

It should be stressed here that the physical relevance of turbulence as an inhibitor of the emergence of mesoscale structures is very dubious. The two-fluid model employed here is derived by averaging over the spatial and temporal resolution employed in the computational setup. The meso-scale structures then appear when employing the two-fluid model with adequate resolution; that is, when using a resolution that is significantly finer than the meso-scales. Such a numerical simulation presupposes that the velocities used in (eqs (1) and (3)) are obtained with the same resolution as the volume fraction fields. A RANS-based turbulence model, on the other hand, is derived by averaging out all of the turbulent velocity fluctuations. Combining the two-fluid model with the Standard $k-\varepsilon$ model therefore results in an attempt to solve for fluctuations in the volume fraction fields without accounting for the fluctuating components of the phase velocities. Consequently, RANS-based turbulence modeling can only be considered compatible with the two-fluid model if the flow is steady or quasi-steady (i.e. there is a clear separation of scales between the mean flow variations and the turbulence and two-phase intermittency) (Bestion 2012).

It is interesting to relate the current results to those obtained when applying some variant of a two-fluid model together with a RANS-based turbulence model to a bubble plume in a flat geometry (Sokolichin and Eigenberger 1999; Pfleger et al. 1999; Mudde and Simonin 1999). If the depth of such a domain is neglected and the geometry described as two-dimensional, the turbulent viscosity becomes much too high and the unsteady motion of the plume is suppressed entirely. In three-dimensional simulations of the thin geometry, the effective viscosity however decreases and a dynamic behavior can be recovered. Of specific interest here is the fact that Sokolichin and Eigenberger (1999), who did not use a full two-fluid model, obtained good agreement 
with the experimental data of Becker, Sokolichin, and Eigenberger (1994), whereas Mudde and Simonin (1999), who used a single-pressure two-fluid model, obtained close agreement with the same experimental data only after incorporating the effect of virtual mass into the interfacial momentum transfer term. These results are very interesting in the light of the fact that the virtual mass terms are generally small but affect the hyperbolicity, and hence the stability, of the two-fluid model employed (Lahey et al. 1980).

In summary, employing the Standard $k-\varepsilon$ model to take the effects of turbulence into account produced high effective viscosities that dampened out the occurrence of meso-scale structures. This numerical experiment highlights the difficulties in describing turbulent two-phase flow using a combination of averaged mathematical models (i.e. a two-fluid model and a RANS model) that are averaged on different length scales. In a mesh refinement study of such a model, small-scale fluctuations in the volume fraction fields that are permitted by the model as such are prevented to appear, as the corresponding velocity field cannot be retrieved. In effect, the correlated fluctuations between the dispersed phase volume fraction field and the continuous phase stress gradient are neglected in the momentum exchange (Igci et al. 2008). The details of the interaction between turbulence and the dispersed phase meso-scale structures must therefore be studied using more comprehensive mathematical frameworks. If meso-scale structures are deemed important for the overall behavior of bubbly flows in nuclear reactors, more work will be needed in the derivation of models for the unresolved fluctuations and their cross-correlations. It is to be expected that more sophisticated turbulence models will be necessary for such simulations (e.g. LES or possibly URANS). It is therefore interesting to note that such turbulence models do not in general work well with dissipative discretization schemes, as these tend to dampen out the resolved turbulent fluctuations and therefore limit the accuracy of the underlying subgrid-scale model. Such issues, which are manageable in single-phase flows, will continue to pose challenges to simulations of gas-liquid flows with a two-fluid model until the complex boundaries and interplay between physical and unphysical instabilities are fully understood.

\section{Conclusions}

In the present work, we formulate a simplistic two-fluid model for the bubbly steam-water flow existing between the fuel pins in nuclear assemblies. The appearance of non-uniform volume fraction fields is investigated and shown to be a function of the bubble loading as well as the domain size. The combination of the two-fluid model with a RANS-based turbulence model is shown to dampen the instabilities and to prevent the non-uniform fields from emerging.

In conclusion, the findings in this work highlight the importance of a consistent filtering approach in the treatment of the volume fraction field and the turbulence in simulations of two-phase flows in nuclear reactors.

Funding: This research was conducted with funding from the Swedish Research Council (Vetenskapsrådet) as a part of the Development of Revolutionary and Accurate Methods for Safety Analyses of Future and Existing Reactors (DREAM4SAFER) framework grant (contract number C0467701). The computations were partly performed on resources at Chalmers Centre for Computational Science and Engineering (C3SE) provided by the Swedish National Infrastructure for Computing (SNIC).

\section{References}

1. Agrawal, K., Loezos, P.N., Syamlal, M., Sundaresan, S., 2001. The role of meso-scale structures in rapid gas-solid flows. Journal of Fluid Mechanics 445, 151-185.

2. Anderson, K., Sundaresan, S., Jackson, R., 1995. Instabilities and the formation of bubbles in fluidized beds. Journal of Fluid Mechanics 303, 327-66.

3. Anglart, H., Nylund, O., Kurul, N., Podowski, M.Z., 1997. CFD prediction of flow and phase distribution in fuel assemblies with spacers. Nuclear Engineering and Design 177, 215-228.

4. Arai, M., 1980. Characteristics and stability analyses for twophase flow equation systems with viscous terms. Nuclear Science and Engineering 74, 77-83.

5. Becker, S., Sokolichin, A., Eigenberger, G., 1994. Gas-liquid flow in bubble columns and loop reactors: PART II. Comparison of detailed experiments and flow simulations. Chemical Engineering Science 49, 5747-5762.

6. Benyahia, S., Sundaresan, S., 2012. Do we need sub-grid scale corrections for both continuum and discrete gas-particle flow models? Powder Technology 220, 2-6.

7. Bestion, D., 2012. Applicability of two-phase CFD to nuclear reactor thermohydraulics and elaboration of best practice guidelines. Nuclear Engineering and Design 253, 311-321.

8. Coquel, F., El Amine, K., Godlewski, E., Perthame, B., Rascle, P., 1997. A numerical method using upwind schemes for the resolution of two-phase flows. Journal of Computational Physics 136, 272-88.

9. Demazière, C., 2013. Multi-physics modelling of nuclear reactors: current practices in a nutshell. International Journal of Nuclear Energy Science and Technology 7, 288-318. 
10. Dinh, T.N., Nourgaliev, R.R., Theofanous, T.G., 2003. Understanding he ill-posed two-fluid model. Proceedings of the $10^{\text {th }}$ International Topical Meeting on Nuclear Reactor Thermal Hydraulics NURETH-10), Seoul, Korea.

11. Esmaeeli, A., Tryggvason, G., 1998. Direct numerical simulation of bubbly flows. Part 1. Low Reynolds number arrays. Journal of Fluid Mechanics 377, 313-345.

12. Gudmundsson, R.L., 2005. A numerical study of the two-fluid models for dispersed two-phase flow. PhD thesis, KTH Royal Institute of Technology, Stockholm, Sweden.

13. Igci, Y., Andrews, A.T. IV, Sundaresan, S., Pannala, S., O'Brien, T., 2008. Filtered two-fluid models for fluidized gas-particle suspensions. AIChE Journal 54, 1431-1448.

14. Ishii, M., Mishima, K., 1984. Two-fluid model and hydrodynamic constitutive relations. Nuclear Engineering and Design $82,107-126$.

15. Joshi, J.B., Deshpande, N.S., Dinkar, M., Phanikumar, D.V., 2001. Hydrodynamic stability of multiphase reactors. Advances in Chemical Engineering 26, 1-130.

16. Keyfitz, B.L., 2001. Mathematical properties of nonhyperbolic models for incompressible two-phase flow. Proceedings of the $4^{\text {th }}$ International Conference on Multiphase Flow, New Orleans, USA.

17. Kreiss, H.-O., Yström, J., 2002. Parabolic problems which are ill-posed in the zero dissipation limit. Mathematical and Computer Modelling 35, 1271-1295.

18. Lahey, R.T. Jr. Cheng, L.Y., Drew, D.A., Flaherty, J.E., 1980. The effect of virtual mass on the numerical stability of accelerating two-phase flows. International Journal of Multiphase Flow 6, 281-294.

19. Launder, B.E., Spalding, D.B., 1972. Lectures in Mathematical Models of Turbulence, Academic Press, London.

20. Lucas, D., Krepper, E., Prasser, H.-M., Manera, A., 2006. Investigations on the stability of the flow characteristics in a bubble column. Chemical Engineering \& Technology 29, 1066-1072.

21. Lucas, D., Prasser, H.-M., Manera, A., 2005. Influence of the lift force on the stability of a bubble column. Chemical Engineering Science 60, 3609-3619.

22. Monahan, S.M., Fox, R.O., 2007. Linear stability analysis of a two-fluid model for air-water bubble columns. Chemical Engineering Science 62, 3159-3177.

23. Morsi, S.A., Alexander, A.J., 1972. An investigation of particle trajectories in two-phase flow systems. Journal of Fluid Mechanics 55, 193-208.

24. Mudde, R.F., Harteveld, W.K., van den Akker, H.E.A., 2009. Uniform flow in bubble columns. Industrial \& Engineering Chemistry Research 48, 148-158.

25. Mudde, R. F., Simonin, O., 1999. Two- and three-dimensional simulations of a bubble plume using a two-fluid model. Chemical Engineering Science 54, 5061-5069.

26. Ojima, S., Hayashi, K., Hosokawa, S., Tomiyama, A., 2014. Distributions of void fraction and liquid velocity in airwater bubble column. International Journal of Multiphase Flow 67, 111-121.

27. Pfleger, D., Gomes, S., Gilbert, N., Wagner, H.-G., 1999. Hydrodynamic simulations of laboratory scale bubble columns fundamental studies of the Eulerian-Eulerian modelling approach. Chemical Engineering Science 54, 5091-5099.
28. Pokharna, H., Mori, M., Ransom, V.H., 1997. Regularization of two-phase flow models: a comparison between numerical and differential approaches. Journal of Computational Physics 134, 282-295.

29. Prosperetti, A., 2003. Two-fluid modelling and averaged equations. Multiphase Science and Technology 15, 181-192.

30. Prosperetti, A., Satrape, J.V., 1990. Stability of two-phase flow models, in: Joseph, D.D. et al. (Eds.), Two Phase Flows and Waves 98-117, Springer-Verlag, New York.

31. Ransom, V.H., Hicks, D.L., 1984. Hyperbolic two-pressure models for two-phase flow. Journal of Computational Physics 53, 124-151.

32. Ransom, V.H., Hicks, D.L., 1988. Hyperbolic two-pressure models for two-phase flow revisited. Journal of Computational Physics 75, 498-504.

33. Sankaranarayanan, K., Shan, X., Kevrekidis, I.G., Sundaresan, S., 2002. Analysis of drag and virtual mass forces in bubbly suspensions using an implicit formulation of the Lattice Boltzmann method. Journal of Fluid Mechanics 452, 61-96.

34. Sankaranarayanan, K., Sundaresan, S., 2002. Lift force in bubbly suspensions. Chemical Engineering Science 57, 3521-3542.

35. Sokolichin, A., Eigenberger, G., 1999. Applicability of the standard $k-\varepsilon$ turbulence model to the dynamic simulation of bubble columns: Part I. Detailed numerical simulations. Chemical Engineering Science 54, 2273-2284.

36. Stewart, H.B., 1979. Stability of two-phase flow calculation using two-fluid models. Journal of Computational Physics 33, 259-270.

37. Stewart, H.B., 1986. On the mathematics of multifield flow. Journal of Mathematical Analysis and Applications 114, 241-251.

38. Toumi, I., 1996. An upwind numerical method for two-fluid two-phase flow models. Nuclear Science and Engineering 123, 147-168.

39. Ustinenko, V., Samigulin, M., loilev, A., Lo, S., Tentner, A., Lychagin, A., Razin, A., Girin, V., Vanyukov, Ye., 2008. Validation of CFD-BWR, a new two-phase computational fluid dynamics model for boiling water reactor analysis. Nuclear Engineering and Design 238, 660-670.

40. Vasquez, S.A., Ivanov, V.A., 2000. A phase coupled method for solving multiphase problems on unstructured meshes. Proceedings of ASME FEDSM'00, ASME 2000 Fluids Engineering Division Summer Meeting, Boston.

41. Wang, J., van der Hoef, M.A., Kuipers, J.A.M., 2009. Why the two-fluid model fails to predict the bed expansion characteristics of Geldart A particles in gas-fluidized beds: a tentative answer. Chemical Engineering Science 64, 622-625.

42. Yang, N., Wu, Z., Chen, J., Wang, Y., Li, J., 2011. Multi-scale analysis of gas-liquid interaction and CFD simulation of gasliquid flow in bubble columns. Chemical Engineering Science 66, 3212-3222.

43. Yström, J., 2001. On two-fluid equations for dispersed incompressible two-phase flow. Computing and Visualization in Science 4, 125-135.

44. Zhang, D.Z., VanderHeyden, W.B., 2001. High-resolution threedimensional numerical simulation of a circulating fluidized bed. Powder Technology 116, 133-141. 